Jap. J. M. Sc. \& Biol., 17, 223-233, 1964

\title{
SOME CONSIDERATIONS ON THE PATHOGENESIS OF HUMAN ASCARIS AND HOOKWORM INFECTIONS : A REVIEW
}

\author{
YOSHITAKA KOMIYA AND TOSHIO YANAGISAWA \\ Department of Parasitology, National Institute of Health, Tokyo
}

(Received: September 2nd, 1964)

\section{Pathogenesis of Human Ascariasis}

Human ascariasis produces a wide variety of symptoms: lassitude, fever, anorexia, dyspepsia, morbid appetite (geophagia), nausea, vomiting, diarrhoea, constipation, abdominal pain, coughing, headache, disorder of consciousness, spasm, asthma, nasal pruritis, visual disturbance, pollakiuria, urticarial rash, etc., which affect nearly all the organs and tissues of the digestive, respiratory, circulatory and excretory systems, and occasionally cause systemic complaints. These symptoms may be attributed to a toxic substance excreted or secreted by worms in the host, a secondary infection with pyogenic bacteria and viruses, mechanical stimulation due to worm movement and allergic reactions of the host to the worms. Extensive studies on the so-called "Ascaris toxin" were made by Koizumi et al. (1944) which unfortunately fell far short of demonstrating satisfactorily that the worm substances and their culture media were involved in the development of ascariasis symptoms. In recent years, the secondary infection has been the subject of a few reports but many points still remain to be clarified.

As is known, clinically symptomless cases of Ascaris infection occur frequently. Some are also described as suddenly presenting marked and serious symptoms, these being exclusively attributed to migration of adult Ascaris in ectopic foci. On the other hand, a growing interest has recently been shown in Japan in the immunological study of parasitic helminths and helminthiasis. Some workers have attempted to elucidate the allergic mechanisms causing manifestations of helminthiasis by comparing them with human allergic reactions to worms.

Two series of investigations will be discussed below. One deals with damage caused by adult Ascaris migrating into ectopic foci in the human abdomen (the "surgical type" of ascariasis, described by Maki, 1958), the other with gastroduodenal ascariasis considered as being one of the allergic manifestations of various forms of ascariasis (Matsumura, 1958). These investigations were chosen because the former is concerned with the type of ascariasis producing acute and severe symptoms and requiring immediate surgical intervention, and the latter because it relates to a new etiology.

a) Pathological and clinical aspects of ascariasis due to migration of adult worms, especially in the abdomen

The fact that the normal habitat of Ascaris in the human host is the upper part

小宮義孝・柳沢十四男（国立予防衛生研究所寄生虫部） 
of the small intestine is generally accepted, while it is well known that the presence of Ascaris in abnormal foci quite frequently provokes marked and violent symptoms due to the movement of the worms. According to Maki (1961) who has reviewed Japanese literature on clinical cases presenting migration of Ascaris, more than 90 per cent of the 1,398 cases investigated had Ascaris in the abdomen, of which about 90 per cent were in the liver, bile-duct, pancreas and appendix (Table 1). This would indicate that

Table 1. A summary of reported cases of adult Ascaris migration in Japan (after Maki, 1961)

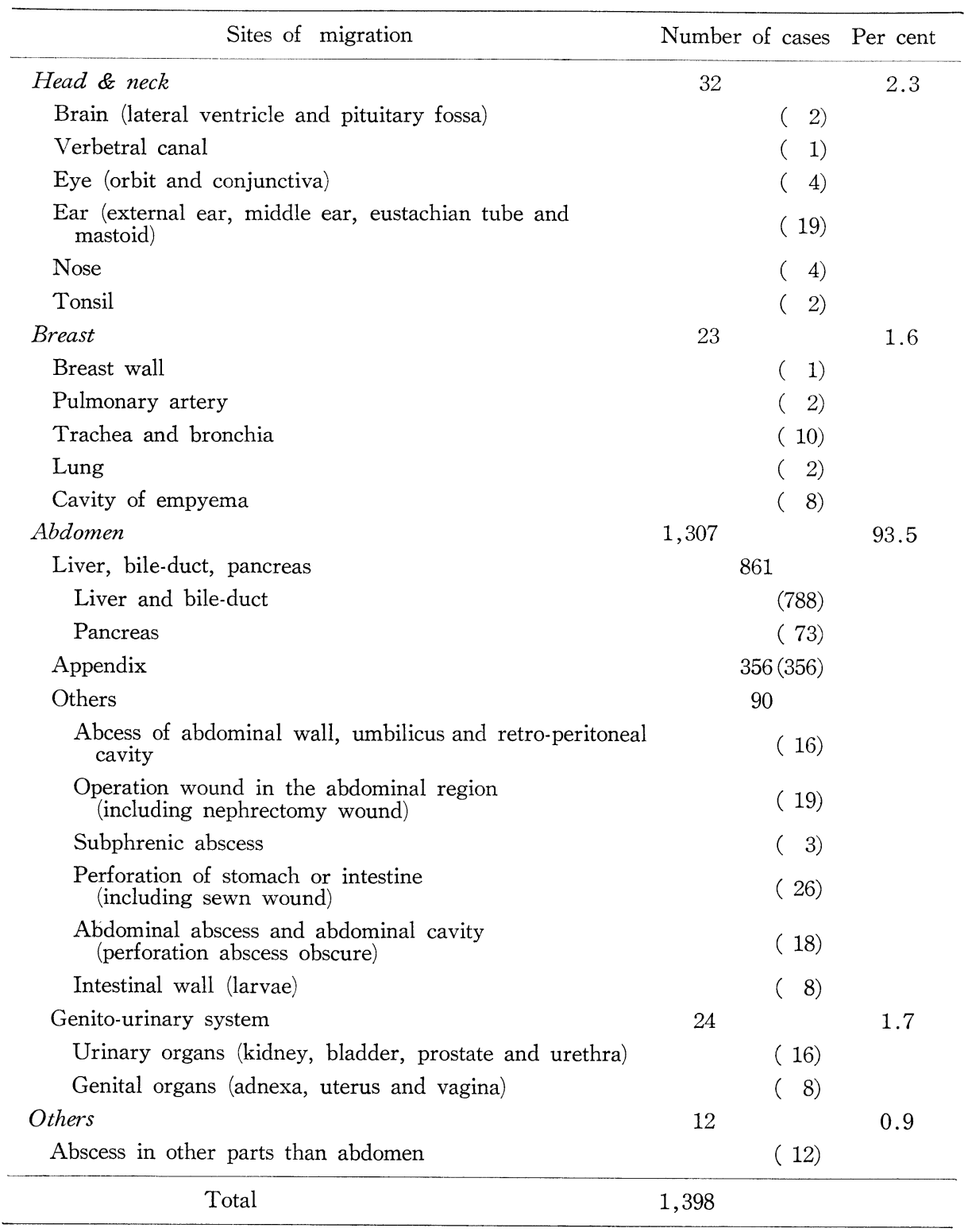


Ascaris migration in these organs amounts to more than 80 per cent of all migrations in the body, although such a high proportion may be due to the proximity of the organs invaded.

1. Bile-duct ascariasis: This condition has been known for some time as being one of the most important that produce symptoms simulating cholelithiasis in Japan (Katsurada, 1908; Nagao, 1901; Uchida, 1903; Yoshida, 1908) as well as in other countries. According to Hashimoto (1954) who reviewed this disease from cases reported in Japan between 1899 and 1952, 242 cases were observed during the period 1899-1945 and 213 between 1946-1952. This might partly reflect a close relationship between the occurrence of the disease and the high post-war incidence of Ascaris infection in Japan, but it might also be due to improved diagnostic methods making early detection possible. Thus Hashimoto assumed that the disease probably occurred frequently in highly endemic areas where it had been previously diagnosed as "stomach spasm".

Various inconclusive interpretations have been made of the mechanism of migration into the bile-duct through the ampulla of Vater: the worms may be forced to leave the bowel due to an unfavourable environment caused by increased acidity, the presence of alcohol and anthelmintics, abdomial cooling and accelerated intestinal peristalsis following high fever or gastro-intestinal catarrh. It has also been maintained that the passing of gallstones through the papilla of Vater may induce worms to migrate to the bile-duct. However, Maki (1955) did not hold with this hypothesis, his clinical records showing that there was no correlation between the occurrence of gallstones and that of worms entering the bile-ducts. He stated that this might be caused by a sort of invasion of worms into a small pit.

Maki (1961) reported that 38 per cent (52 cases) of 138 uncomplicated cases with bile-duct ascariasis presented with a history of violent colic in the epigastric region, simulating "stomach spasm", and that 15 out of 59 patients gave a history of gastralgia. Generally speaking the patients complained of sudden colicky pain in the subcostal region and at other times in the substernal region, lasting from a few days to 10 days. Nausea and vomiting developed in more than 80 per cent of the cases showing gastralgia, but Ascaris were seldom vomited. A low temperature $-37^{\circ} \mathrm{C}$ - was usually noted but it rose to as much as $38^{\circ} \mathrm{C}$ or more when cholangitis and/or cholecystitis occurred as intercurrent diseases. Two interesting cases reported by Igarashi (1940) and Karasawa (1951) showed no icterus, although 34 and 29 worms were counted in the bile-duct respectively. In the early stages of bile-duct ascariasis infection there are marked subjective symptoms such as colicky pain, whilst objective symptoms are very few. A commonly observed subjective symptom is that of violent pain when pressing the substernal region; this was reported as having occurred in 92 per cent of 54 cases investigated by Maki (1961).

2. Cholelithiasis of Ascaris origin: Dead and living Ascaris in the bile-duct may cause gallstone formation due to inflammatory substances in the bile juice, eggs laid and fragments of the worm cuticle. This is supported by the fact that substances and fragments of Ascaris origin have been detected in bilirubin calcium stones which characterize Japanese cholelithiasis, contrary to the cholesterol nature of this condition in Europeans and Americans. Yasuda (1955) using an improved technique for the detection of Ascaris elements in gallstones, reported that these were found in 33 out of 64 cases examined and in 28 out of 55 bilirubin gallstones collected in the hospital in Aomori Prefecture. This report suggests that cholelithiasis is closely associated with Ascaris infection in Japan. The pathogenesis, pathology and symptomatology of this type of 
cholelithiasis resemble those of non-parasitic cholelithiasis. It is, therefore, not considered necessary to discuss them in this paper.

3. Appendiceal ascariasis: Genuine parasitic appendicitis may be caused exclusively by Ascaris migrating into the lumen of the appendix. However, it is rather difficult to diagnose real worm appendicitis due to the high incidence of both Ascaris infection and appendicitis in Japan. In order to determine the relationship between appendicitis and Ascaris migration, several surveys were made on patients operated for appendicitis. Ascaris was found in 17 out of 2,168 cases (Fukuchi, 1941), 2 out of 388 (Watanabe, 1939), 7 out of 174 (Miyamoto, 1953), 15 out of 268 (Tsushima, 1949), and 7 out of 584 (Maki, 1961). A sudden colicky pain in the right iliac region may be considered a clinical symptom of worms in the appendix, whilst objective symptoms tend to be rather mild in nature.

The mechanism of migration of Ascaris into the human appendicular lumen was studied by Miyamoto (1953). According to his very interesting report, six out of eight cases with parasitic appendicitis suffered from colicky pain, presumably due to the sudden abnormal movement of Ascaris in the lumen immediately following the administration of Santonin and kainic acid preparations. A similar movement of worms produced by anthelmintics, observed on the X-ray film, was also reported by Makidono (1957). These clinical observations suggest that Ascaris may not infrequently migrate into the appendix when anthelminthics are given. Consequently, the administration of anthelmintics may play an important role in starting the migration of Ascaris:

4. Pancreatic ascariasis: Ascaris seldom wander in the pancreatic tube (Yokota, 1947). During a period of four years after the Second World War 29 cases with acute pancreatitis were, however, reported by Maki (1950) who stated that only three of all the cases treated for acute pancreatitis had no history of ascariasis, and that 16 out of 26 cases having a history had vomited Ascaris, the remaining cases having passed worms per anum. It may, therefore, be assumed that the incidence of acute pancreatitis due to Ascaris migrating in the pancreatic tube is high in areas where ascariasis is endemic. Acute parasitic pancreatitis was considered to be caused by Ascaris wandering into both the bile and the pancreatic ducts, thus forcing pancreatic juice activated by duodenal juice back into the pancreatic duct. In Europe and America, acute pancreatitis is a common complication of cholelithiasis (50-78 per cent of cases seen by McClure \& Jankelson, 1946). Few cases presenting this complication have, however, been reported in Japan. This difference may be considered to be due to the frequent occurrence in Japan of acute pancreatitis caused by wandering Ascaris (Maki, 1961).

The symptom of acute pancreatitis is sudden and growing colicky pain in the left hypochondriac region. At times such pain abates within a few hours, but if it persists there is nausea and vomiting which result in a worsening of the condition.

Chronic pancreatitis may be caused by recurrent acute forms of pancreatitis, or by Ascaris eggs scattered in the pancreatic parenchyma. On the other hand, chronic pancreatitis may sometimes be a sequel of cholelithiasis or cholangitis, as suggested by Miyake (1952) who reported 94 complicated cases with pancreatic inflammation out of 543 cases with cholelithiasis. Manifest clinical symptoms of parasitic pancreatitis are: bulging subcostal area, fever and leukocytosis, the latter being less frequent. Most of the patients had a history of epigastric colic simulating bile-duct ascariasis.

b) Allergic manifestations in gastroduodenal ascariasis

The possibility of allergic reactions being closely related to the manifestations of 
ascariasis was suggested by Miyagawa (1957), who stated that human allergic reactions to Ascaris corresponded to a wide variety of ascariasis symptoms. Some of these symptoms, especially those connected with the digestive organs and nervous system - urticarial rash, colicky pain resulting from Ascaris migrating to ectopic foci in the visceral organs. spasm produced by intestinal obstruction (ileus) due to masses of writhing worms - may be suspected as being related to the host's allergic reactions to worms.

In Japan, studies on allergy to Ascaris originated from the work on the so-called "Ascaris toxin" started by Koizumi et al. (1944) who reported that Ascaris perienteric fluid contained a histamine-like substance causing marked allergic reactions in the human skin and mucous membranes. Later, studies were conducted on the allergenicity of Ascaris body and its culture media, utilizing the improved techniques and progress made in the field of immunology - skin reaction, Shwartzman's reaction, Arthus phenomenon, and Prausnitz-Küstner reaction - with a view to collecting the evidence of antigenicity of the various fractions of the worm body and its culture media extracts (Ikeda, 1956; Morishita, 1953, 1956 ; Komiyama, 1954 ; Matsumura, 1956 ; Nakajima, 1954 ; Miura, 1950: Yamamoto, 1954). These workers were concerned with the demonstration of allergenicity in the worms but not with the direct interpretation of clinical manifestations in human ascariasis. Although some workers succeeded in inducing urticaria in the human skin by the administration of certin substances contained in the worms, thus providing proof of their allergenicity, they could not always demonstrate a direct link with the manifestations of ascariasis. Recently Matsumura (1956) reported that some symptoms seen in gastroduodenal ascariasis were caused by the host's allergic reactions. He commenced his experiment with guinea-pigs entirely non-sensitized to Ascaris, which were laboratory-reared, care having been taken to prevent any Ascaris infection. Ascaris-sensitized and non-sensitized guinea-pigs were simultaneously given Ascaris-enteric fluid orally. This experiment showed that marked erythema, congestion, petechial haemorrhage and erosion occurred in the gastroduodenal mucosa of sensitized guinea-pigs but not in the non-sensitized ones. The perienteric fluid used in this experiment showed positive Schultz-Dale reaction and, furthermore, induced spasms of the stomach, as well as of the small intestine in the sensitized guinea-pigs. Spasms in the stomach and intestine of sensitized guinea-pigs were well inhibited by anti-allergic substances such as adrenaline and antihistamines. On the other hand, no appreciable change was observed in non-sensitized animals. The same was observed when the animals were given the medium in which Ascaris had been cultivated. This indicates the occurrence of a common antigenic substance in perienteric fluid and culture media, and further, leads to the hypothesis that when the antibody is produced in the human stomach and intestine sensitized to Ascaris infection, an antigenic substance from Ascaris will react with the antibody, resulting in inflammatory changes in the host's mucosa and simultaneously in the development of colicky pain, the gastrointestinal spasm being an allergic reaction to worms. Matsumura's hypothesis was supported by results obtained from his elaborate clinical experiments on humans : when a person with a recent history of gastroduodenal ascariasis was orally given culture media together with a barium meal, clinical symptoms simulating those of gastroduodenal ascariasis - colic and pain on pressing the epigastric region, nausea and vomiting - developed. Under X-ray examination, it was observed that an increased peristalsis in the stomach and intestine, and pyloric stenosis lasting about six hours occurred and responded to ephedrine and antihistamine treatment. The mechanism underlying the manifestations of gastroduodenal ascariasis - one of many forms of ascariasis - was thus elucidated from the viewpoint of allergy by Matsumura 
(1956). Nearly all the other symptoms developing in ascariasis still remain to be clarified by the further study of the possible allergic processes involved.

\section{Pathogenesis of Human Hookworm Infection}

Many clinical parasitologists recognize that the most common subjective and objective symptoms found in hookworm patients are connected with the circulatory system (palpitation), the nervous system (dizziness, headache, tinnitus, etc.) and other systemic symptoms such as lassitude and anasarca. The objective symptoms observed on clinical examination include anaemia, enlargement of the heart, abnormal cardiac sound. These findings indicate that kookworm disease is one of the typical helminthiasis inducing parasitic anaemia.

\section{a) Etiology of hookworm anaemia}

The nature and cause of hookworm anaemia has been the object of numerous publications during the last forty years, but the mechanism involved in the development of anaemia is still debated. Two theories as to its causation are advanced: one being the so-called "blood-loss theory of anaemia with iron deficiency", in which the anaemia is attributed to loss of blood through the sucking action of the parasites; the other, the so-called "toxin theory" which emphasizes the role of a toxin produced by the parasite in.addition to blood loss. The former theory, supported by the American school, originated from the works of Wells (1931) and Nishi (1934) who made a quantitative study of the amount of blood sucked from the dog's intestinal wall by the canine hookworm. These were later supplemented by the works of Suerez (1933), Cruz (1934), Road et al. (1934), Foster (1936), etc. who reported that hookworm anaemia readily yielded to iron therapy. The latter theory was postulated largely by Japanese parasitologists, Miyagawa et al. (1933), Kitayama et al. (1950), Eguchi (1950), Miyoshi (1952), Noda (1950), Katada (1954) and others.

\section{Theory of hookworm anaemia due to blood loss with iron deficiency}

(i) Amount of blood loss: According to Wells (1931) who observed canine hookworms in dogs' intestines, worms attached to the intestinal wall sucked blood at the rate of 120-150 suctions per minute, with the result that the blood filled the worm's intestinal lumen so fully that it was finally expelled per anum. The amount of blood ingested by a single worm per day was calculated to be as much as $0.8 \mathrm{ml}$ on the basis of the number of red cells collected in 20 minutes in a calibrated pipette placed at the posterior part of the worms, it being assumed that the same rate of suction continued during a 24-hour period. In Japan Nishi (1934) made a similar observation. He stated that the amount of blood excreted through the anus by a single worm per day was estimated to average $0.144 \mathrm{ml}$, with a maximum of $0.268 \mathrm{ml}$ on the basis of the frequency of blood discharged through the anus (1.36 times per minute). His observation showed $0.2 \mathrm{ml}$ blood seepage around the buccal cavity of a single worm per day, and a total daily blocd loss per worm of $0.36 \mathrm{ml}$ with a $0.7 \mathrm{ml}$ maximum. Almost the same amount (0.384) of daily blood loss through canine hookworm was also reported by Liang (1937). However, Foster et al. (1954) did not agree with such high figures of daily blood loss, which in their opinion was around $0.1 \mathrm{ml}$. A daily blood loss through Ancylostoma duodenale and Necator americanus amounting to $0.12-0.2$ and $0.03-0.05 \mathrm{ml}$ respectively was recently reported by Masuya (1958), who determined the values of haemoglobin and the related haem pigment, pyridin ferrochromogen, by means of the Flink-Watson method. Masuya's findings (1958) are roughly in agreement with those of Roche et al. (1957). Using 
radioactive chromium $\mathrm{Cr}^{51}$, these authors found that blood loss per day per $N$. americanus averaged $0.0311 \mathrm{ml}$ with a standard deviation of 0.0173 (range : $0.0118-0.0635 \mathrm{ml}$ ) and that the quantity lost per $A$. duodenale was in the region of $0.2 \mathrm{ml}$. It appears that the theory of hookworm anaemia caused by blocd loss associated with iron deficiency is well supported by all these studies. Recently Foy and Nelson (1963) reviewing the literatures on anaemias commonly encountered in the tropical countries, concluded that iron deficiency anemia was usually assocoated with blood loss due to hookworm infection.

(ii) Experimental induction of anaemia in dogs by frequent controlled haemorrhages: Foster et al. (1934) induced anaemia experimentally in dogs by withdrawing blood from them and compared it with that of dogs infected with canine hookworm. Their work revealed that anaemia induced by venesection and hookworm infection anaemia were very alike and that both responded to iron therapy. They came to the conclusion that there was no disturbance in the haematopoietic function of the bone marrow in the case of canine hookworm anaemia and, furthermore, that the anaemia was caused by the mechanical blood loss due to worm suction. After iron treatment, recovery from human hookworm anaemia was somewhat slow according to Saurez (1933), Cruz (1934), and Road et al. (1934) who stated that the anaemia was not primary, therefore haematopoiesis in the bone marrow was not affected, but that it was instead of a microcytic and hypochromic nature similar to anaemia induced by blood loss with iron deficiency. It followed that hookworm anaemia may be caused by an iron deficiency due to loss of blood, insufficient iron supply and/or a lowered absorption capacity of the host's intestine.

(iii) Blood loss theory in Japan: Increased numbers of erythroblasts and eosinophil leukocytes were demonstrated in hookworm cases by Japanese workers (Komiya, 1930 ; Nakajima, 1951; Kitayama et al., 1951 ; Masuya, 1958 ; Saruta, 1959 ; Oda, 1953). This haemotological finding indicated the nature of the anaemia to be hypochromic or iron-deficient. Some of these workers thought that it suggested an enhanced haematopoietic function. The blocd loss theory of hookworm anaemia received early support from many Japanese investigators (Oba, 1929 ; Matsuura, 1931 ; Liang, 1937 ; Shirakawa, 1943 ; Iwata et al., 1947 ; Morita, 1948 ; Eguchi et al., 1950) who believed that hookworm anaemia in the early stages of infection might be caused by a loss of blood and, in the later stages, by a toxin released by the parasites. A clinical observation of 34 uncomplicated cases of hookworm infection, conducted by Nakao et al. (1953) showed an increase in the number of blood cells and especially of odd-shaped erythroblasts which returned rapidly to normal when treatment with iron preparations was instituted both in wormed and non-wormed cases. Iron therapy did not appear to have any appreciable effect on the erythrocytes in both groups of patients. Nakao et al. finally came to the conclusion that the main cause of hookworm anaemia was either insufficiency of iron available for the host's haematopoiesis or abnormal iron metabolism.

Further justification of the blood loss theory was furnished by Masuya (1958). He defined hookworm anaemia as being a strictly typical anaemia presenting all the characteristics of iron-deficiency. Having observed that the hookworm patients he had treated had a microcytic and hypochromic blood picture, he put forward the idea that no primary hyperchromic anaemia suggested the presence of a toxin. In an attempt to interpret the causation of hookworm anaemia, a quantitative determination in the patient's stools of the blood loss due to hookworm was carried out by the Flink-Watson method. The figures were two to $22 \mathrm{ml}$ of blood loss, corresponding to $11 \mathrm{mg}$ iron per day. If one considers that only $1 \mathrm{mg}$ iron is cleared in a healthy adult per day, it is easy to 
understand the considerable disturbance such a blood loss causes in the iron metabolism of hookworm patients. Masuya offered another proof of anaemia due to iron deficiency: the more frequent cccurrence of anaemia in females than in males. The fact that the haemoglobin level and serum iron values determined in a group of factory workers positive for eggs were higher than in other hookworm patients may be easily explained: from the bill of fare over the past six months their daily iron intake was estimated to amount to as much as $17 \mathrm{mg}$, i. e. 1.7 times more than the average iron intake of the Japanese population.

As is known, some doubts have been expressed regarding the theory of hookworm anaemia due to blood loss; anaemia was thought to be caused by disturbances in the host's iron absorption (Fukushima, 1950), by the unavailability of iron stored in the liver (Kometani, 1955), or by the partial retention of iron in the reticulo-endothelial system (Kitajima, 1957). But Masuya observed no difference in the quantity of serum iron between infected and non-infected people who were given iron preparations, no difference in absorptive function when they were given liver or reduced-iron preparations, but an absence in the patients of iron in the bone marrow and liver. He thus concluded that hookworm anaemia is caused by a failure in iron metabolism due to iron deficiency resulting either from blood loss or from insufficient iron intake of the host.

\section{2. "Toxin theory" of hookworm anaemia}

The idea that a toxic substance released by the parasites in the host's intestine is probably, together with intestinal blood loss, an important etiological agent of hookworm anaemia, has been upheld for quite a time by many workers in Japan and other countries: Inada (1909), Narita (1926), Hosono (1923), Hayashi (1909), Petri (1908), Wippel (1909) and Weinberg (1913) who demonstrated the presence of a haemolytic lipoidal toxin causing human hookworm anaemia and cccurring both in the hookwoom and in the serum of hookworm patients. Later, however, this substance was proved to be so widely distributed in many types of animal tissues such as those of Ascaris, Schistosoma japonicum, the mucosa of the human digestive tract, spleen and liver, that many workers have now given up the theory because of its non-specificity for hookworms.

Miyagawa et al. (1933) experimenting on dogs suggested that toxin might play an important role in the development of anaemia associated with hookworm infection : a marked anaemia was seen in heavily infected dogs as early as three or four to seven days following inoculation and, when dissected, the spleen was seen to be clearly enlarged (twice the normal size). This confirmed that there was anaemia before the worms reached the host's intestine and sucked blood; therefore, it might be produced by a disturbance in the host's haematopoiesis effected by a toxin (or a metabolite) from the parasites.

The toxin theory of hookworm anaemia thus advocated by Miyagawa et al. (1933) was further expanded on, which was due in part to the detection of an anaemia-inducing substance in the worms as well as in the culture media in which they were maintained and the infected host, and in part to the determination of the physicochemical proporties of this substance. An anaemia-producing substance was demonstrated in culture media by Fujita (1955), Shibata (1954), Matsumoto (1955, 1956, 1957), Iwata (1961), Suzuki (1961), etc. Fujita (1955) succeeded in developing a marked anaemia in rabbits by giving them culture medium, non-infected dog serum supplemented by haemolysed blood cells in which adult and larval hookworms had been maintained, or the ether-soluble substance, while nothing happened in the control rabbits. Iwata (1961) and Suzuki 
(1960) using fourth- and fifth-stage larvae proved the presence in culture media of a substance producing anaemia. Such a substance was also found in the parasite body by many Japanese workers (Ueno, 1928; Takahashi et al., 1938 ; Komori, 1943 ; Miura, 1950 ; Matsumoto, 1952 ; Sugawa, 1954 ; Imawari, 1956 ; Takayanagi, 1957, and others). Kitayama and his co-workers reported that a higher ability to produce the anaemia was shown in the worm extracts soluble in fatty solvents as compared with those in the residue. They also demonstrated a higher ability in female worms, particularly the blood picture of the laboratory animals used was not indicated. Kitada (1954) obtained a substance in the gastric juice of hookworm patients which was soluble in methanol, non-precipitable in a trichloracetic acid solution and produced anaemia in rabbits when five to six intravenous injections of $2-3 \mathrm{ml}$ extract were given every second or third day (RBC : 60 per cent ; $\mathrm{Hb}: 65$ per cent). Kasahara (1953) established the presence of this substance in the cerebrospinal fluid, milk and urine of patients and suggested that it was absorbed and circulated in the host's body.

As mentioned above, not all partisans of the toxin theory rejected other possible causative factors of anaemia. Whilst strongly emphasizing the important role of the worm-toxin in the development of hookworm anaemia to account for the early appearance of anaemia in experimentally infected dogs, i.e. before worms reach the intestine and suck blood, they were more or less willing to accept the proposal that intestinal blood loss was one of the causes of anaemia. Miyagawa (1956) for instance stated that anaemic hosts showed an acute infectious enlargement of the spleen and that heavily infected cases frequently presented leukocytosis in the early stage of infection, but developed afterwards primary anaemia with marked leukopenia.

On the contrary, adepts of the blocd loss theory in Japan as elsewhere, on the basis of experiments and clinical examinations, maintain that hookworm anaemia is microcytic and hypochromic and of the serum iron deficiency type which responds well to iron therapy. It may then be very possible that two different avenues of investigation have led to two different theories. Although, as pointed out by Masuya (1956), haematological data have been insufficiently reported in studies designed to establish the worm toxin as a cause of anaemia, Schretzenmayer (1938), Kitakawa et al. (1950) as well as Miyagawa (1956) have described the marked primary anaemia with its characteristic blood picture in heavily infected cases and in the advanced stage of infection. Here the question arises as to whether hookworm anaemia is primary or of the iron deficiency type, but unfortunately no theory has yet been proposed which could settle this point. Apart from the question relating to the validity of the two existing theories on the genesis of hookworm anaemia, the nature of the anaemia should first be investigated in detail before one or the other interpretation can be considered as reflecting the truth.

b) Causation of some symptoms in hookworm disease

1. Causation of spoon nail and morbid appetite in hookworm patients: As indicated above, the most common symptoms of hookworm disease are the systemic symptoms and those pertaining to the circulatory, nervous and digestive systems. The first three groups of symptoms may be definitely attributed, directly or indirectly, to anaemia. Causation of morbid appetite like geophagia and of spoon nail is still debated, although the former is presumably due to vagotonia caused by intoxication, and the latter to malnutrition. In this connexion, Masuya (1958) made very interesting observations. He reported that after venesection dogs showed a sharp morbid appetite for toilet paper and cotton-wool, and that the typical spoon nail of an aged anaemic female patient who 
daily lost as much as $63 \mathrm{ml}$ of blood in feces as a result of stomach cancer, disappeared after iron therapy had been instituted. Thus these symptoms, previously considered as being due to other factors, were clearly proved to be caused by iron-deficiency anaemia.

2. Allergical manifestations of hookworm disease: The mechanism producing symptoms of the digestive organs has not yet been elucidated. Recently Ishizaki et al. (1960) analysed hookworm disease manifestations from the viewpoint of allergy. They conducted a series of parallel tests (skin test with hookworm antigen-sensitivity and related tests - histamine, eosinophilia, cholinesterase activity in serum) in hookworm carriers in order to establish correlation between an allergic constitutional disposition and manifestations of hookworm disease. A positive correlation between skin sensitivity to histamine and skin sensitivity to hookworm antigen injected was observed in hookworm carriers. At the same time a significant negative correlation between the diameter of the wheal produced by hookworm antigen and cholinesterase activity in the serum was also confirmed in carriers. The eosinophil count in patients was modified as anticipated: the number of eosinophils in the patients increased in proportion to the diameter of erythema around the skin reaction. Systemic symptoms such as lassitude, vertigo, stiff shoulder and others such as abdominal pain, diarrhoea and a coated tongue were also correlated with positive skin tests. Symptoms of the circulatory system such as palpitation, cold feet, abnormal cardiac sound, occurred more frequently in carriers showing a high sensitivity to hookworm antigen. Ishizaki et al. thus came to the conclusion that the allergic reaction may play an important role in the manifestations of hookworm disease, since the allergic constitution, as determined by the skin test, appears to be closely related to the subjective symptoms developed in hookworm infection.

Although the allergenicity of hookworm extract had been demonstrated before by many Japanese workers (Morishita, 1956; Ishihara, 1956 ; Suzuki, 1954) and the socalled "ground itch" and "Wakana disease"* in Japan ascribed to allergenic agents, Ishizaki et al. (1961) succeeded in determining the interrelationship between hookworm allergen and some manifestations of hookworm disease.

\section{REFERENCES}

FAUST, E. C. (1949): Human helminthology, 3rd ed., Philadelphia, Lea \& Febiger, p. 744.

Faust, E. C. \& Russel, P. F. (1957) : Clinical parasitology, 6th ed., Philadelphia, Lea \& Febiger, p. 1078.

FOY, H. \& NELSON, G. S. (1963): Helminths in the etiology of anemia in the tropics, with special reference to hookworms and schistosomes, Parasitological Reviews. Exptl. Parasitology, $14,240-262$.

IKEDA, M. (1956): Ascaris allergy: Symposium on parasite allergy, Kiseichyugaku Zasshi, $5(2)$, 294. (text in Japanese).

Ishizaki, T., Kutsumi, H., Kumada, M., Komiya, Y., Araki, H., Takayama, H., Okada, K. \& NozAKI, S. (1961): Clinical studies on hookworm carriers VII. Allergological aspect of the symptoms produced by hookworm infection, Kiseichyugaku Zasshi, 1O(2), 211-220. (text in Japanese with English summary)

KoIZumi, M. (1944): Studies on Ascaris, 1st et., Tokyo, Dainihon-insatsu Kabushiki-kaisha. (text in Japanese)

KomIYA, Y. (1958): Hookworm and hookworm disease, 1st ed., Tokyo, Sekibundo, p. 93. (text in Japanese)

KOMIYA, Y. (1959): The cause of hookworm anemia, its effect and treatment. The recent

* "Wakana" is a young leaves of Chinese cabbage in Japanese. 
trend of parasite studies, Igakuno-doko, 23, 73-93. (text in Japanese)

MAKI, T. \& MiURA, F. (1947) : Acute pancreatitis and Ascaris infection, Sogoigaku, 4, 125129. (text in Japanese)

Maki, T. \& Akimoto, T. (1949): Sogoigaku, 6, 1076-1078. (text in Japanese)

MAKI, T. (1956): Bile-duct ascariasis: Symposium on ascariasis, Kiseichyugaku Zasshi, 5 (2), 287-288. (text in Japanese)

MAKI, T. (1961) : The surgical type of ascariasis. In : Achievement in Japanese parasitology, Tokyo, Ishiyakushuppan K. K., 1, 250-278. (text in Japanese)

MASUYA, T. (1958): The pathophysiology of hookworm disease, Kiseichyugaku Zasshi, 7 (3), 304-306. (text in Japanese)

Matsumura, T. \& InOue, K. (1951): Problems on gastroduodenal ascariasis, Rinsho-naikashonika, 6. (text in Japanese).

MAtsumurA, T. (1956): Gastroduodenal ascariasis: Symposium on ascariasis, Kiseichyugaku Zasshi, 5 (2), 288-289. (text in Japanese)

Miyagawa, Y., IshiI, S. \& SHIMIZU, S. (1933) : Experimental studies on hookworm anemia, I, II \& III, Jikken-igaku Zasshi, 17, 1155-1172, 1173-1193. (text in Japanese)

MrYAGAWA, Y. (1959): Clinical consideration on parasite allergy. The recent trend of parasite studies, Igakuno-doko, 23, 129-146. (text in Japanese)

MryagaWA, Y. (1956) : Clinical helminthology. In : Helminthiasis 1st ed., Tokyo, Chyugaiigakusha, 1, 311. (text in Japanese)

Mryagawa, Y. (1957): In: Helminthiasis, 1st ed., Tokyo, Chyugai-igakusha, 2, 395. (text in Japanese)

MoRISHITA, T. (1956): Hookworm allergy: Symposium on parasite allergy, Kiseichyugaku Zasshi, 5 (2), 295-296. (text in Japanese)

NakaO, K., Shima, M., Toyokura, Y., Kinugasa, K., Maekawa, T. \& Omori, T. (1953) : On the hookworm anemia with special reference to its statistical consideration, Nihon Naikagakkai Zasshi, 42, 23-30.

NisHI, M. (1933) : Experimental studies on the causation of hookworm anemia, Taiwan-igaku Kai Zasshi, 32, 677-691. (text in Japanese with English summary).

Wells, R. S. (1931): Observation on blood-sucking activity of the hookworm, Ancylostoma duodenale, J. Parasitol., 17, 167-182. 